\title{
地下管线普查工作中不同阶段的质量控制
}

\author{
宋鹤宁 \\ 沐城测绘(北京)有限公司
}

DOI:10.32629/gmsm.v3i1.479

[摘 要] 城市地下管线是现代化城市的重要组成部分, 在城市建设和运维中扮演者重要的角色,承担着非常重要的责任。地下管线普查是为掌 握管线信息而进行的测绘工作,而管线普查的质量直接影响到城市以后的规划设计是否准确,还影响到城市管线突发事件的应急响应处置的效 率。本文针对地下管线普查工作的流程,对前期准备和生产过程中的质量控制措施做了一定阐述,对于过程质量控制的重要性做详细论述,仅供 读者参考。同时也思考了井下实景三维数据在管线系统中应用的前景。

[关键词] 地下管线; 普查; 过程质量控制

\section{1 过程质量控制方法和监督抽查内容}

1. 1 过程质量控制方法

地下管线普查项目部应建立并运行完善的质量管理体系, 设立专门的 质量管理和质量检查机构, 配备专职质量检查人员, 加强过程工序质量控 制, 建立质量岗位责任制, 落实质量责任。作业承担单位应组织专职质量检 查人员对地下管线普查工作进行全过程质量检查, 重点检查质量管理体系 运行情况和质量控制效果, 对发现的问题及时归纳分析, 提出整改意见, 并 监督整改落实 ${ }^{[1]}$ 。

\section{2 监督抽查内容}

管线普查工作分为不同的流程阶段: 前期准备、生产过程、成果验收 等, 针对不同阶段的过程进行质量抽查。

\section{2 前期准备工作内容及质量控制}

2. 1 前期准备工作内容

地下管线普查工作的前期准备包括资料收集、现场踏勘、探测仪器方 法试验、仪器一致性校验、技术设计书编制、技术交底等工作流程 ${ }^{[2]}$ 。

\section{2 资料收集过程质量控制}

管线普查工作范围确定后, 项目承接单位应收集已有管线资料、测区 基本比例尺地形图、高等级控制点成果资料 ${ }^{[3]}$ 。通过项目委托单位联系测 区内各管线权属单位收集测区内已有管线的现状资料, 包括管线竣工图、 设计图、施工图等。

(1) 管线资料收集以竣工图为主, 以保证资料的准确性。资料内容包括 管径、材质、建设年代和权属单位等信息。(2) 地形图作为管线普查的底 图, 其现势性影响外业管线草图的编制和外业的工作效率。(3)测区控制点 分布应均匀合理, 坐标与高程系统满足项目要求。

\section{3 现场踏勘过程质量控制}

踏勘时对收集的管线资料与现场管线对比核查是否一致, 核查收集的控 制点位置及保存情况，了解地下管线的种类、密度、材质、干扰情况等信息。

\section{4方法试验过程质量控制}

进行方法试验的目的是为技术设计书的编制提供数据依据。应在编制 技术文件之前进行方法试验, 如有新方法应用时必须进行方法试验。方法 试验选择测区内最有代表性的管线进行, 进行方法试验的人员应具有管线 探测的经验, 试验内容应根据地下管线的埋设方式、深度、管线材质及管 线接口类型确定。确定探测方法后编制方法试验报告。

\section{5仪器一致性校验质量控制}

不同的探测仪器在相同的地理条件、相同的传输频率和传输功率下具 有不同的场强。为了观察上述条件下不同仪器的勘探结果是否一致, 为保 证检测结果的准确性, 应在检验开始前对每台仪器进行一致性检验。验证内
容包括定位和深度一致性检查。校准工作已在调查区域中进行, 已获取了地 下管线数据。仪器校准应使用相同的检测方法和相同的工作参数, 重复检测 同一位置的管道, 定位和深度确定结果应一致或相对误差不超过 $5 \%$ 。

管线仪的校验应符合下列规定:

(1) 在一条已知管线上, 选择一种信号施加方式, 采用相近的工作频 率、发射功率和收发距探查地下管线的平面位置和埋深; (2) 记录仪器探 查的平面位置与实际平面位置的差值, 探查的深度与管线实际深度差值。

通过与已知地下管线数据比较或通过开挖验证, 以保证仪器的精度, 保证投入普查工作的每台仪器在精度上具有一致性。隐蔽管线点的定位、 定深精度度符合下表要求:

表1 隐蔽点精度

\begin{tabular}{|c|c|c|}
\hline 管线中心埋深 & 水平位置限差 $\delta \mathrm{ts}$ & 埋深限差 $\delta \mathrm{th}$ \\
\hline $\mathrm{h} \leqslant 1000$ & $\pm 100 \mathrm{~mm}$ & $\pm 150 \mathrm{~mm}$ \\
\hline $\mathrm{h}>1000$ & $\pm 0.10 \mathrm{~h}$ & $\pm 0.15 \mathrm{~h}$ \\
\hline
\end{tabular}

注: 表中 $\mathrm{h}$ 为管线中心埋深, 单位为毫米。

地下管线探测仪校验后通过记录数据计算得到的定位均方差不应大 于水平位置限差的 $1 / 3$, 定深均方差不应大于埋深限差的 $1 / 3$ 。

$$
\begin{aligned}
& \text { 仪器一致性定位均方差: } \mathrm{M}_{\mathrm{ks} \mathrm{s}}= \pm \sqrt{\sum_{i=1}^{n} \Delta S i^{2} / \mathrm{m}-\mathrm{n}} \\
& \text { 仪器一致性定深均方差: } \mathrm{M}_{\mathrm{tsh}}= \pm \sqrt{\sum_{i=1}^{n} \Delta \mathrm{hi} \mathrm{i}^{2} / \mathrm{m}-\mathrm{n}}
\end{aligned}
$$

式中:

$\triangle S$ 一探测位置与实际位置的误差; $\triangle \mathrm{h}$ 一探测埋深与实际埋深的误 差; $m$ 一总观测次数, 即参加校验的所有点上全部观测次数之和; $n$ 一参与 校验的点数。

校验工作结束后, 编制探测仪器一致性校验报告。在实际普查项目实 施中严格控制不合格仪器、未经校验不得投入项目使用。

\section{3 生产过程中的质量控制}

3.1控制测量过程质量控制

3.1.1平面控制测量过程质量控制。随着GPS技术的应用普及, 目前全国 大部分省市都布设了连续运行基准站 (CORS) 系统, 完全满足日常测量工作的 控制要求。测绘过程中严格按照操作规程布设、观测, 观测应符合以下规定:

(1) 仪器观测的收玫阈值设置, 平面坐标不应超过 $2 \mathrm{~cm}$, 垂直坐标不应 超过 $3 \mathrm{~cm}$ 。(2) RTK观测应符合下列规定：观测前仪器进行初始化；观测值 应为RTK固定解且PDOP值小于 6 时采集的数据; 自动观测不应少于 10 个历 元; 经纬度应记录到 0.00001 “, 三维坐标应记录到0.001m。(3)测回间仪 器重新进行初始化, 两测回时间间隔应大于1分钟。(4)测回间的平面坐标 
较差不应超过 $2 \mathrm{~cm}$, 垂直坐标较差不应超过 $3 \mathrm{~cm}$, 应取平均值作为最终成果。 (5) RTK控制测量应符合下列规定：控制点应布设不少于 3 个并保证两相邻 点通视; 控制点测量应采用三角支架方式架设天线进行作业, 测量过程中 仪器的圆气泡应严格稳定居中。

控制点应采用常规方法进行边长、角度或导线联测检核, 检核测量技 术要求应符合下表规定:

表2 控制点检核技术要求

\begin{tabular}{|c|c|c|c|c|c|c|}
\hline \multirow[b]{2}{*}{ 等级 } & \multicolumn{2}{|c|}{ 边长检核 } & \multicolumn{2}{|c|}{ 角度检核 } & \multicolumn{2}{|c|}{ 导线联测检核 } \\
\hline & $\begin{array}{c}\text { 测距中误差 } \\
(\mathrm{mm})\end{array}$ & $\begin{array}{c}\text { 边长较差相对 } \\
\text { 中误差 }\end{array}$ & $\begin{array}{c}\text { 测角中误差 } \\
(")\end{array}$ & $\begin{array}{c}\text { 角度较差限差 } \\
(\text { (") }\end{array}$ & $\begin{array}{c}\text { 角度闭合差 } \\
(")\end{array}$ & $\begin{array}{c}\text { 边长相对闭 } \\
\text { 合差 }\end{array}$ \\
\hline 一级 & 15 & $1 / 14000$ & 5 & 14 & ${ }_{16} \sqrt{n}$ & $1 / 10000$ \\
\hline 二级 & 15 & $1 / 7000$ & 8 & 20 & $24^{\sqrt{n}}$ & $1 / 6000$ \\
\hline 三级 & 15 & $1 / 4000$ & 12 & 30 & ${ }_{40} \sqrt{n}$ & $1 / 4000$ \\
\hline 图根 & 20 & $1 / 2500$ & 20 & 60 & $60^{\sqrt{n}}$ & $1 / 2000$ \\
\hline
\end{tabular}

3. 1. 2 高程控制测量过程质量控制。核查项目设计书保证高程系统的 采用符合项目要求, 采用符合项目、规范要求的高程起算点, 并分析起算点 的分布是否合理。

检查水准仪、标尺等是否符合水准测量的要求, 保证仪器检定在有效 期内, 检查水准仪 $\mathrm{i}$ 角是否符合要求。水准测量时避开中午时段观测, 避免 大气折光的影响, 保证记录清晰不得涂改, 需改正时把数字划线改正, 观 测、记录等人员须签字。

采用专业的平差软件对水准数据进行平差, 水准路线闭合差限差不应 大于 $10 \sqrt{n} \mathrm{~mm}$ ( $\mathrm{n}$ 为测站数, $\mathrm{n} \leqslant 50$ ), 线路总长不应超过 $8 \mathrm{~km}$; 控制点稀少地 区可同级附合 1 次。平差计算需要换人进行重新计算平差, 两次结果进行对 比分析, 保证数据的准确性。

\section{2 数据采集质量控制}

3. 2. 1明显点数据采集质量控制。现场巡视注意探查人员和仪器设备的 变动情况, 检查调查范围和管线的取舍是否符合设计要求, 检查管线点设置 的完整性、正确性, 检查管线点调查属性的完整性、正确性, 检查外业记录 的及时性和真实性。开井调查时, 量测人员报量测数据后记录人员须重复数 据, 无误后记入记录表, 当管井内有堆积物或淤泥时, 须使用排水杆扞插管 线, 再量取排水杆的深度, 保证量测数据的准确性。量测的埋深、管块等数 据建议用石笔在井壁上记录, 以备后续检查复核。明显点复核测量时埋深的 粗差率不超过 $5 \%$, 错误率不超过 $2.5 \%$, 且粗差率和错误率之和不超过 $5 \%$ 。

3.2.2 隐蔽点探测质量控制。管线隐蔽点是管井之间不可见的变向、 变径、变深点, 需要通过管线探测仪或探地雷达探测才可定位的管线点, 由于其不可见性, 隐蔽点的探测需要具有一定工作经验的探测人员主导探 查, 项目工作期间固定探查人员, 且管线探测仪也必须是经过仪器一致性 校验合格的仪器, 当探测人员和仪器发生变化时, 及时做人员技术验证和 仪器的一致性校验, 通过后方可开展探查工作。

隐蔽点开挖验证时实地测量管线的平面位置和埋深, 与探测数据进行 对比, 查验管线的相关属性是否正确。隐蔽管线点重复探查时要实地探查 管线的平面位置和埋深, 平面位置或埋深的粗差率不超过 $7.5 \%$, 平面位置 或埋深的错误率不超过 $5 \%$, 且粗差率和错误率之和不超过 $7.5 \%$, 隐蔽管线 点开挖验证的超差率不超过 $10 \%$ 。超差率计算公式:

超差率 $=\frac{\text { 开挖验证超差点数 }}{\text { 开挖验证总点数 }} \times 100 \%$

3. 2.3 点位测量过程质量控制。目前碎部点位测量的主要方法有RTK
测量和全站仪测量, 但由于地下管线位于城市道路中, 道路两侧高大建筑 物较多, RTK的使用具有局限性, 无法达到精度要求, 全站仪采集仍是当下 主流的测绘方法。

全站仪采集碎部点的主要误差来源有仪器对中误差、目标偏心差、仪 器误差、外界环境的影响等。加强操作人员的技术培训提高技术水平, 使 用正式的控制点成果资料, 设站时做第三点检核, 使用年检合格的仪器进 行测绘, 管线明显点、隐蔽点做明显标记防止漏测、串号等。

3. 3 内业处理过程质量控制

管线的内业处理是以外业草图为依据以专业入库软件为平台, 实现从 纸质记录到电子数据的录入过程。数据库录入完成后, 不同作业组间进行 $100 \%$ 互检, 内业负责人的抽检比例不得少于 $30 \%$ 。检查数据的合理性与完整 性: 按范围检查管线属性表的埋深数据是否在合理范围内; 管线点的特征 和附属物与数据字典的一致性; 点号编码、代码与编码规则的一致性; 埋 设方式与数据字典的一致性; 管径和材质的有效性; 道路名称或代码有效 性; 压力与电压有效性; 权属单位名称代码有效性; 排水流向的合理性; 管块孔的总数与管线占用孔数是否合乎逻辑; 坐标、高程值范围的合理性; 超长管线检查; 测量范围全覆盖完整性、属性字段完整性检查等。数据 处理质量控制由人机交互和软件辅助完成, 做到流程同步数据全覆盖。

3. 4 图形编辑质量控制

管线图的编制按照国家或地方标准规定, 不同管线采用不同颜色设计, 管线图设计背景要以城市地形为依据。

管线图形编绘过程中的质量控制包括数据格式、定位基准、比例尺、 分幅编号、图幅整饰和图面要素的完整、正确、规范、一致性、逻辑性质 量控制。

管线图编辑完成后, 需要对管线的接边位置进行检查, 对于不同测区、 不同图幅、不同组的接边位置重点检查, 检查数据的一致性及数据存储的 咒余, 从而保证整个区域管线图形的连通。需进行管线图形叠加分析, 根据 外业普查成果数据生成矢量管线图, 同外业组的管线图进行叠加分析, 检 查成果数据与管线图是否存在误差。

\section{4 结束语}

地下管线是 “城市的生命线”, 地下管线的普查工作是建设现代化城 市的必要工作, 地下管线普查的数据质量关乎人民生命安全, 优质的管线 成果数据和完善的地下管网系统, 才能为城市发展、规划和管理提供科学 的依据。普查过程中注意每个环节的关键问题, 从地下管线的外业普查、 数据的生产, 到数据库的建立, 引入全过程的质检机制, 对项目实施全程的 的质量控制, 严把质量关。以上是笔者通过对地下管线的普查工作, 对地下 管线普查数据质量控制的一些总结和认识, 并提一下自己的建议, 目前城 市地下管线系统虽建立了三维管线系统, 但大部分为以数据构建的三维模 型, 不能直观反映井下的实际情况, 能否以三维扫描实景代替? 如果可以 实现的话, 虽说对硬件和软件系统的需求进一步提高, 但也将是管线普查 工作质的飞跃。

\section{[参考文献]}

[1]林广元.浅谈城市地下管线普查与管理[J].北京测绘,2005,(1):59-60+48. [2]江贻芳.地下管线探查作业的质量控制[J].北京测绘,2006,(1):47-50. [3]肖絮飞.城市地下管线数字化流程[J].北京测绘,2004,(1):37-39+42.

作者简介：

宋鹤宁 $(1981--)$, 男, 河北保定人, 汉族, 本科, 工程师, 从事工程测量 工作。 